The truth of the final statement of the theorem emerges when to $k$ are assigned successively the values $1,2, \cdots$, $m-1$, and when it is recalled that a sufficient condition that the manifold defined by the equations $(A)$ has no singular points in $R$ is that the matrix $M_{m}$ is of rank $m$ at all points of the manifold in $R$.

HARVARD UNIVERSITY

\title{
ON THE FOUNDATIONS OF GENERAL INFINITESIMAL GEOMETRY*
}

BY HERMANN WEYL

In connection with a seminar on infinitesimal geometry in Princeton, in which I took part, it seemed desirable to clarify the relations between the work of the Princeton school and that of Cartan.

With a group (f) of transformations in $m$ variables $\xi$ is associated, in accordance with Klein's Erlanger Program, a homogeneous or plane space $\Re$ of the kind ( 3 ; a point of $\Re$ is represented by a set of values of the "coordinates" $\xi^{\alpha}$ and figures which go into each other on subjecting the coordinates to a transformation of $(5)$ are to be considered as fully equivalent. The transformations of $(5)$ give at the same time the transition between two allowable "normal" coordinate systems in $\Re$. If we have two spaces $\Re, \Re^{\prime}$ of the kind $(\$)$ and set up a definite normal coordinate system in each of them, then such a transformation can be interpreted as an isomorphic representation of $\Re$ on $\Re^{\prime}$. (S) is assumed to be transitive.

Cartan $\dagger$ developed a general scheme of infinitesimal geometry in which Klein's notions were applied to the tangent plane and not to the $n$-dimensional manifold $M$ itself. The

* Presented to the Society, June 21, 1929

$\dagger$ E. Cartan, Sur les variêtés à connexion affine et la théorie de la relativité généralisée, Annales de l'École Normal Supérieure, vol. 40 (1923), pp. 325412 ; in particular, p. 383 , etc. 
object of his work can be briefly described as follows. There is associated with each point $P$ of $M$ an $m$-dimensional plane space of the kind $\$$. To the transition from $P$ to a neighboring point $P^{\prime}$, that is, to the line element $P P^{\prime}$, corresponds an isomorphic representation of $T_{P}$ on $T_{P^{\prime}}$, the "displacement" $T_{P} \rightarrow T_{P^{\prime}}$ or briefly $\overline{P P^{\prime}}$. On this basis the introduction of a general concept of curvature is possible: if we displace the tangent plane $T_{P}$ in $P$ along a curve $L$ on $M$ which leads back to $P$ the tangent plane returns in a new position or orientation. The final position is obtained from the original by a certain isomorphic representation of $T_{P}$ onto itself, and this we call the "curvature along $L . "$

For the purpose of analytic formulation we refer $M$ to coordinates $x^{i}$ and introduce a normal coordinate system $\xi^{\alpha}$ in each tangent plane $T_{P}$. Let the components of $\overline{P P}^{\prime}$ be $d x^{i}$. We assume that the $\xi^{\alpha}$ of all the $T_{P}$ can be so chosen that the displacement $P P^{\prime}$ is an infinitesimal isomorphic representation of the same order of magnitude as the $d x^{i}$. A radical specialization is introduced by the further assumption that this displacement depends linearly on $\overline{P P^{\prime}}$, i.e. that the consecutive application of the displacements $P P^{\prime}$ and $P^{\prime} P^{\prime \prime}$ shall yield the displacement $P P^{\prime \prime}$. The displacement from $P$ to all neighboring points $P^{\prime}$ is then expressed by the formula

$$
d \xi^{\alpha}=-\sum_{i} u_{i}^{\alpha}(\xi) d x^{i}
$$

The curvature along the infinitesimal parallelogram formed by line elements $d x$ and $\delta x$, which consequently has as components

is given by

$$
(\Delta x)^{i k}=d x^{i} \delta x^{k}-\delta x^{i} d x^{k}
$$

$$
\begin{aligned}
\Delta \xi^{\alpha} & =R_{i k}^{\alpha}(\xi) d x^{i} \delta x^{k}=\frac{1}{2} R_{i k}^{\alpha}(\xi)(\Delta x)^{i k} \\
R_{i k}^{\alpha} & =\left(\frac{\partial u_{k}^{\alpha}}{\partial x^{i}}-\frac{\partial u_{i}^{\alpha}}{\partial x^{k}}\right)+\left(\frac{\partial u_{k}^{\alpha}}{\partial \xi^{\rho}} u_{i}{ }^{\rho}-\frac{\partial u_{i}^{\alpha}}{\partial \xi^{\rho}} u_{k}{ }^{\rho}\right) .
\end{aligned}
$$

In the foregoing the tangent plane is not tied up with the manifold; in order to justify this designation and hold to the 
idea of a tangent plane we must now imbed it into the manifold. The first step in this process consists in taking a definite point $O$ of $T_{P}$ as center which shall, by definition, cover the point $P$ on $M$ (imbedment of 0 th order). This leads to a restriction in the choice of a normal coordinate system $\xi$ on $T_{P}$; because of the transitivity of $\&$ it can and shall be so chosen that the coordinates $\xi$ vanish in the center. The group $B$ is restricted to the subgroup $\mathbb{H}_{0}$ of all representations of $B$ which leave the center $O$ invariant. On displacing the tangent plane along a closed curve $L, O$ goes over into a point $O^{*}$ whose deviation from $O$ characterizes the "torsion along $L . " R_{i k}^{\alpha}(0)=0$ is the recessary and sufficient condition that $M$ be without torsion.

The idea of tangent plane further requires that the line elements of $T_{P}$ issuing from $O$ shall "coincide" with the line elements of $M$ issuing from $P$; this correspondence must be a one-to-one affine representation. But having already required imbedment of 0 th order the method of accomplishing this imbedment of 1 st order is fixed. The center $O^{\prime}$ of $T_{P^{\prime}}$ arises by the displacement $P P^{\prime}$ from a definite point $O_{1}$ of $T_{P}$, and we let the line element $\overline{O O}_{1}$ on $T_{P}$ correspond to the line element $\overline{P P^{\prime}}$ on $M$. For purposes of calculation it is, however, more convenient to consider the line element $\overline{O_{1}^{\prime} O^{\prime}}$ on $T_{P^{\prime}}$ which arises from $\overline{O O}_{1}$ by the displacement $P P^{\prime}$. The $\xi$-coordinates of $O_{1}^{\prime}$ on $T_{P^{\prime}}$ are $d \xi^{\alpha}=-u_{i}{ }^{\alpha}(0) d x^{i}$, and consequently

$$
d \xi^{\alpha}=u_{i}{ }^{\alpha}(0) d x^{i}
$$

are the components of the line element ${\overline{O_{1}^{\prime} O_{1}}}_{1}$ on $T_{P^{\prime}}$ or $\overline{O O}_{1}$ on $T_{P}$. The condition that this linear relation between $(d x)$ and $(d \xi)$ be one-to-one reciprocal involves two requirements: (1) the dimensionality $m$ of the tangent plane (which was until now arbitrary) must be the same as the dimensionality $n$ of the manifold $M$, and (2) the determinant $\left|u_{i}^{\alpha}(0)\right| \neq 0$. If (5) contains the affine group, and we shall henceforth assume that it does, the coordinate system $\xi^{\alpha}$ on $T_{P}$ can be further adapted to the given coordinate system $x^{i}$ on $M$ in such a 
way that corresponding line elements shall have the same components : $u_{i}^{\alpha}(0)=\delta_{i}^{\alpha}$.

If $\$$ were the affine group the previous requirements would fully specify the normal coordinate system $\xi^{\alpha}$ on $T_{P}$ in its dependence on the coordinates $x^{i}$ chosen on $M$; but this is not the case if $B$ is a more extensive group. That is, the "tangent plane" $T_{P}$ is not as yet uniquely determined by the nature of $M$, and so long as this is not accomplished we can not say that Cartan's theory deals only with the manifold $M$. Conversely, the tangent plane in $P$ in the ordinary sense, that is, the linear manifold of line elements in $P$, is a centered affine space; its group $(S)$ is not a matter of convention. This has always appeared to me to be a deficiency of the theory; I consider that above all, the infinitesimal-geometric researches of Eisenhart, Veblen, T. Y. Thomas, and others in Princeton $\dagger$ have remedied this blemish for projective and conformal geometry.

The connection between $\xi$ and $x$, although not yet uniquely determined by the previous postulates, allows us to conclude the following: In the development

$$
R_{i k}^{\alpha}(\xi)=R_{i k}^{\alpha}(0)+R_{\beta i k}^{\alpha} \xi^{\beta}+\cdots,
$$

the quantities $R_{i k}^{\alpha}(0)$ and $R_{\beta i k}^{a}$ in the point $P$ are determined by the coordinates $x^{i}$ alone and transform on transformation of coordinates as tensors of order 3 and 4 , of the kind indicated by the position of the indices. It is therefore an invariantive restriction to require that our manifold be such that (1) it is without torsion and (2) $\sum_{\alpha} R_{a i k}^{\alpha}$ vanish; we call such a manifold "special."

Let $\$$ be the projective group. We must then proceed to imbedment of second order in order that $T_{P}$ be completely

$\dagger$ L. P. Eisenhart, Non-Riemannian Geometry, 1927. O. Veblen, Projective tensors and connections, Proceedings of the National Academy, vol. 14 (1928), p. 154; Conformal tensors and connections, ibid., vol. 14 (1928), p. 735. T. Y. Thomas, $A$ projective theory of affinely connected manifolds, Mathematische Zeitschrift, vol. 25 (1926), p. 723. 
determined by $M$. We consider, as an analog, the contact of two surfaces

$$
y=f\left(x^{1}, \cdots, x^{n}\right), \quad y=f^{\prime}\left(x^{1}, \cdots, x^{n}\right)
$$

in $(n+1)$-dimensional space. Let

$$
f^{\prime}-f=a+a_{i} x^{i}+\frac{1}{2} a_{i k} x^{i} x^{k}+\cdots
$$

in the neighborhood of the origin. There is contact of 0 th order (intersection) if $a=0$, of 1 st order (tangency) if in addition the linear terms are not present, $a_{i}=0$, and finally contact of second order (osculation) if further all $a_{i k}$ vanish. I refer to the two surfaces as semi-osculating if in addition to $a$ and $a_{i}$ the sum $\sum_{(i)} a_{i i}$, the spur of the quadratic terms, vanishes. Analogously we demand that $T_{P}$ not only be tangent to the manifold but further that it be semi-osculating. The name tangent plane is then misleading, but we shall use it instead of the more correct "projective semi-osculating plane" for the sake of brevity. The exact definition is as follows. Given an infinitesimal volume element $V$ in $P$, say a parallelepipedon obtained from line elements in $P$ which shall be infinitely small in comparison with $\overline{P P}^{\prime}=\left(d x^{i}\right)$. Let $V^{\prime}$ be the "same" volume element in $P^{\prime}$, that is, it shall be generated from the line elements with the same components in $P^{\prime}$; naturally this construction is dependent on the particular coordinate system employed. Because of the imbedment of order $V$ coincides with a volume element $V$ in $O$ on $T_{P}$ and $V^{\prime}$ with one such in $O^{\prime}$ on $T_{P^{\prime}}$; this latter is obtained from an element $V_{1}$ in $O_{1}$ on $T_{P}$ by the displacement $P P^{\prime}$. We now require that $V$ and $V_{1}$ have the same volume, measured in the coordinates $\xi$ on $T_{P}$.

It is again more convenient for the calculation to take $V$ and $V_{1}$ over into $V_{1}^{\prime}, V^{\prime}$ on $T_{P}$, by means of the displacement $P P^{\prime}$. The isomorphic representation which carries $V^{\prime}$ into $V_{1}^{\prime}$ is by definition simply (1)-taken for $\xi$ 's which are infinitely small compared to the $d x^{i}$. Consequently we write

$$
u_{i}^{\alpha}(\xi)=\delta_{i}{ }^{\alpha}+\Gamma_{\beta i}^{\alpha} \xi^{\beta}
$$


and on introducing

we find

$$
\stackrel{\alpha}{\Gamma_{\beta i} d x^{i}}=d \stackrel{\alpha}{\alpha}
$$

$$
\log \left(V^{\prime} / V_{1}^{\prime}\right)=\log \left(V_{1} / V\right)=\sum_{\rho} d \gamma_{\rho}^{\rho}
$$

Our condition is that this trace shall vanish, and we assert that it can be fulfilled by appropriate choice of the projective coordinates $\xi^{\alpha}$ on $T_{P}$. The previous requirements determine the $\xi$ except for a projective transformation of the type

$$
\bar{\xi}^{i}=\frac{\xi^{i}}{1+\sum \alpha_{k} \xi^{k}},
$$

which leaves the center unaltered and is the identity to terms of first order in the neighborhood of the center. The ratio of the measures of volume elements $V$ and $\bar{V}$ in $\xi$ and $\bar{\xi}$, situated at $\left(\xi^{\alpha}\right)$, is given by the functional determinant

$$
\left|\frac{\partial \bar{\xi}^{i}}{\partial \xi^{j}}\right|=(\bar{\xi} \xi) .
$$

For infinitely small $\xi$ this determinant is

$$
\overline{(\xi} \xi)=1-(n+1) \sum \alpha_{k} \xi^{k} .
$$

Our volume $V$ is at the point $\xi=0, V_{1}$ at $\xi^{\alpha}=d x^{\alpha}$, and consequently

$$
\log \left(\bar{V}_{1} / \bar{V}\right)=\log \left(V_{1} / V\right)-(n+1) \sum \alpha_{i} d x^{i}
$$

from which we see that in order that $\bar{V}_{1}=\bar{V}$ the $\alpha_{i}$ can be chosen in one and only one way; $\alpha_{i}=\Gamma_{\rho i}^{\rho}$.

The projective coordinate system $\xi$ on $T_{P}$ is now completely specified by the coordinates $x^{i}$ on $M$. If we refer $M$ to a new coordinate system $\bar{x}^{i}$ we shall have a new projective coordinate system $\xi$ on $T_{P}$. The projective transformation $\xi \rightarrow \bar{\xi}$ can be described by the facts (1) that it agrees in the neighborhood of the origin with the transformation $x \rightarrow \bar{x}$ in terms of first order about $P$ and (2) that the functional determinant $(\bar{\xi} \xi)$ in 0 agrees with $(\bar{x} x)$ in terms of 1 st as well as 
0th, order. H. P. Robertson* pointed out in a short note that this relation is the decisive point in Veblen's transformation theory of projective space. What we do here, however, is not simply connect the transformations of the $\xi$ with those of $x$ but rather we associate a projective $\xi$ coordinate system on $T_{P}$ with an individual $x$ system on $M$. This possibility arises from the fact that we begin with the projective connection and with its aid tie up the $\xi$ with the $x$, i.e. accomplish the complete imbedment of the tangent plane. But on the other hand the transformation of the $\xi$ is determined by the transformation of the $x$, as described above, without taking the given projective connection into account. Veblen's procedure corresponds to this method: this relation between the two transformations is first obtained and the corresponding invariant theory of possible projective connections then developed.

The introduction of $n+1$ homogeneous projective coordinates $\eta$ by means of the equation $\xi^{i}=\eta^{i} / \eta^{0}$ is for the present purely a matter of convenience. The formulas for the displacement expressed in terms of them have the form

$$
d \eta^{\alpha}=-d \gamma_{\beta}^{\alpha} \eta^{\beta}, \quad d \gamma_{\beta}{ }^{\alpha}=\Gamma_{\beta}{ }^{\alpha} d x^{i} .
$$

(From now on Greek indices shall run from 0 to $n$ and Latin from 1 to $n$.) Since only the ratios of the $\eta$ are to be considered we can and shall introduce the normalizing condition $d \gamma_{0}{ }^{0}=0$. We then have $\Gamma_{\rho i}^{\rho}=\delta_{i}^{\alpha}$ and $\Gamma_{\rho i}^{\rho}=0$. In the case of a special manifold (see above) we have furthermore the symmetry condition $\Gamma_{k i}^{\alpha}=\Gamma_{i k}^{\alpha}$ and the $\Gamma$ with only Latin indices determine the remaining components. This leads to the theorem: If we allow only special manifolds the projective connection is determined uniquely by the geodesics. $\dagger$

* H. P. Robertson, Note on projective coordinates, Proceedings of the National Academy, vol. 14 (1928), p. 153.

$\dagger$ See J. A. Schouten, Rendiconti di Palermo, vol. 50 (1926), pp. 142-169, in particular p. 158. I do not find that this work, which is closely related to our process, gives a clear account of the fact that the coordinates $\xi$ must be tied up with the $x$ as described above. 
For the complete development of projective infinitesimal geometry we must, in my estimation, add three independent ideas to Cartan's scheme; the first and most important of these consists in connecting the coordinates $\xi$ with the $x$ by the requirement of "semi-osculation," the second answers the question to what extent the geodesics determine the projective connection by the invariantive "specialization." The third idea, which I now consider, is due to T. Y. Thomas: it is possible to give the variables $\eta$ themselves, and not only their ratios, a geometrical interpretation. The analytic expression in coordinates $\eta$ for any projective mapping which leaves $O$ invariant,

$$
\bar{\eta}^{i}=\sum_{k} a_{k}{ }^{i} \eta^{k}, \quad \bar{\eta}^{0}=\eta^{0}+\sum_{k} \alpha_{k} \eta^{k}
$$

can be so normalized that the coefficient $a_{0}^{0}=1$. This normalization is useful because it is not destroyed by composition: the group $\left(_{0}\right.$ is replaced by the isomorphic group of affine transformations in $n+1$ dimensions of the form (4). If the transition $\eta \rightarrow \bar{\eta}$ on $T_{P}$ corresponds to the transition $x \rightarrow \bar{x}$ on $M$ the transformation (4) can further be described as agreeing with the transformation of the differentials $d x^{0}, d x^{1}, \cdots, d x^{n}$ in $P$ when the additional coordinate $x^{0}$ transforms in accordance with the law

$$
\bar{x}^{0}=x^{0}-\frac{1}{n+1} \log (\bar{x} x) .
$$

We now have an $(n+1)$-dimensional manifold $M^{*}$ instead of $M$; each point of $M$ is replaced by a filament of $M^{*}$ along which $x^{1}, \cdots, x^{n}$ are constant and only $x^{0}$ varies. By (5) the distance between points on the same filament, i.e. the difference of their $x^{0}$ coordinates, as well as the filaments themselves, have an invariantive significance. An $(n+1)$-dimensional affine tangent plane, the domain of the variables $\eta$, is associated with each point of $M^{*}$. The transformation of the $\eta$, which is related to a transformation of the $x$ on $M$, is the same for all points on the same filament. Extending the $\Gamma$ by adding $\Gamma_{\beta 0}^{\alpha}=\delta_{\beta}^{\alpha}$, this means that the ratios $\eta^{0}: \eta^{1}: \cdots: \eta^{n}$ 
of a point on the tangent plane are unaltered by displacement along the filament. The $n$-dimensional projective displacement on $M^{*}$ defined by

$$
d \eta^{\alpha}=-d \gamma_{\beta}^{\alpha} \eta^{\beta}, \quad d \gamma_{\beta}^{\alpha}=\Gamma_{\beta \rho}^{\rho} d x^{\xi},
$$

is consequently invariantively determined by the projective displacement on $M$.

We must next ask if this is also true for the $(n+1)$ dimensional affine displacement expressed by the same formulas; the answer is affirmative, because our normalization is so chosen that $\Gamma_{\beta_{\rho}}^{\alpha}$ is symmetric in $\beta$ and $\rho$. To show this, let $\bar{\Gamma}_{\beta_{\rho}}^{\alpha}$ be the projective connection of $M$ evaluated in a new coordinate system $\bar{x}^{i}$ in the manner described above, and let $\tilde{\Gamma}_{\beta_{\rho}}^{\alpha}$ be the components of the same affine connection on $M^{*}$ expressed in terms of the coordinates $\bar{x}^{i}$ in the manner indicated by (6) in terms of the $x^{i}$. Then the corresponding equations (6) characterize the same projective connection, that is, $\bar{\Gamma}_{\beta \rho}^{\alpha}$ and $\tilde{\Gamma}_{\beta \rho}^{\alpha}$ can differ only by a term of the form $\delta_{\rho}^{\alpha} \lambda_{\rho}$. Now $\bar{\Gamma}$ as well as $\tilde{\Gamma}$ must be symmetric in the two lower indices and consequently

$$
\delta_{\beta}^{\alpha} \lambda_{\rho}=\delta_{\rho}^{\alpha} \lambda_{\beta},
$$

from which we obtain by the contraction $\alpha=\rho$

$$
\lambda_{\beta}=(n+1) \lambda_{\beta}, \quad \lambda_{\beta}=0 .
$$

All that we have said here can be taken over mutatis mutandis to the conformal geometry. Here the equation

$$
g_{i k} d x^{i} d x^{k}=0
$$

on $M$ is fundamental. If $g_{i k}$ be the values of the coefficients in $P$ then the conformal geometry on the homogeneous "tangent" plane $T_{P}$ is described by the equation $g_{i k} d \xi^{i} d \xi^{k}=0$ with constant coefficients $g_{i k}$; the group (s) consists of all transformations of the $\xi$ which leave this equation invariant and consequently depends on the point $P$ in question. The conformal displacement $P P^{\prime}$ must be a transformation which takes the equation $g_{i k} d \xi^{i} d \xi^{k}=0$ over into $g_{i k}^{\prime} d \xi^{\prime i} d \xi^{\prime k}=0$. Consequently this does not agree literally with the scheme de- 
veloped above-furthermore (5) does not contain the affine group (only the orthogonal one). We must expressly require that the coincidence of the line elements $\overline{P P}^{\prime}$ on $M$ and $\overline{O O}_{1}^{\prime}$ on $T_{P}$ is a conformal, and not merely an affine, relation. (1) The requirement of semi-osculation is also here sufficient to tie up the conformal coordinate system $\xi$ uniquely with the $x$. (2) If the manifold is special the conformal connection is uniquely determined by equation (7). (3) The transition to homogeneous coordinates $\eta$, in which the conformal representation appears as a homogeneous linear transformation, is here accomplished, following Möbius, by

$$
\eta^{0}: \eta^{1}: \cdots: \eta^{n}: \eta=1: \xi^{1}: \cdots: \xi^{n}:-\frac{1}{2} g_{i k} \xi^{i \xi} \xi^{k} .
$$

These coordinates are subject to the relation

$$
g_{i k} \eta^{i} \eta^{k}+2 \eta^{0} \eta=0 \text {. }
$$

It is convenient for the purpose of calculation to normalize the coefficients $g_{i k}$, only the ratios of which are given, by the condition $\left|g_{i k}\right|=1$. This third and last step, which was carried through by Veblen in a recent paper, proceeds as before, but the result is more complicated since we have $n+2$ variables $\eta$, whereas Thomas' extension gives but $n+1$ coordinates $x$. Consequently we do not arrive at an affine connection on an $(n+1)$ - or $(n+2)$-dimensional manifold $M^{*}$, invariantly related to the conformal connection on $M$.

Princeton University 